\title{
ARTICLE
}

\section{Benzodiazepines for anxiety disorders: maximising the benefits and minimising the risks ${ }^{\dagger}$}

\author{
Vladan Starcevic
}

Vladan Starcevic is an Associate Professor at the University of Sydney and Sydney Medical School. He is Head of the Academic Department of Psychiatry at Nepean Clinical School and consultant psychiatrist at Nepean Hospital in the western suburbs of Sydney. His main professional and research interests include psychopathology, psychopharmacology and psychotherapy of anxiety, mood, somatoform and personality disorders; combined pharmacotherapy and psychotherapy; and psychiatric classifications. Dr Starcevic serves on the panel for the World Federation of Societies of Biological Psychiatry Task Force on Treatment Guidelines for Anxiety, Obsessive-Compulsive and Posttraumatic Stress Disorders.

Correspondence Dr Vladan Starcevic, Nepean Hospital, Department of Psychiatry,

PO Box 53, Penrith NSW 2751,

Australia. Email: vladan.starcevic@ sydney.edu.au

†For a commentary on this article see pp. 259-262, this issue.

\begin{abstract}
SUMMARY
Benzodiazepines still play an important role in the management of anxiety disorders but dependence is associated with their therapeutic use. The key to effective and safe long-term use of benzodiazepines is: the careful selection of patients who might benefit from them; administration in clinical situations in which they are more likely to be beneficial; use of lower doses and in conjunction with an antidepressant, if possible; monitoring and managing their side-effects; and minimising the risk of withdrawal symptoms and relapse, mainly through tapering the dose and/or combining with effective psychological interventions.
\end{abstract}

\section{DECLARATION OF INTEREST}

V.S. has received honoraria for talks at meetings sponsored by Lundbeck, the Lundbeck Institute and AstraZeneca, and travel assistance from AstraZeneca, Boehringer Ingelheim and the Lundbeck Institute.

The use of benzodiazepines for the treatment of anxiety disorders has long been controversial. It has divided doctors into those who emphasise problems with their use and avoid them, and those who continue to prescribe them, albeit reluctantly and even secretively. Complicating matters further, some doctors express one opinion about benzodiazepines publicly and quite the opposite view privately. Benzodiazepines are also a common reason for the rift between patients who believe that they are useful and doctors who refuse to prescribe them because of their 'addictiveness'. This has produced confusion in both physicians and patients. The aim of this article is to provide a balanced appraisal of the role of benzodiazepines in the treatment of anxiety disorders, emphasising how to make the most of these drugs.

\section{History and current use of benzodiazepines}

Benzodiazepines - the prototypical anxiolytics were introduced in the early 1960s. They quickly became very popular for a variety of reasons. The most succinct explanation for their rise was the societal need for substances with calming effects. Over the centuries this need has been met mainly with alcohol. In the decade prior to the introduction of benzodiazepines, barbiturates and meprobamate were often used to alleviate anxiety and distress. However, these medications were associated with dependence, and barbiturates were lethal in overdose. Being much safer than barbiturates and meprobamate, benzodiazepines were described as 'one of the twentieth century's greatest inventions' (Healy 2002). Small wonder, then, that in the mid-1970s they became the most widely prescribed group of psychotropic medications in the world (Balter 1974).

What caused the subsequent fall in enthusiasm for benzodiazepines? It is undoubtedly the fact that they are associated with pharmacological dependence and that difficulties in ceasing the drugs after long-term use are common. One inquiry into this matter concluded that addiction should be differentiated from non-addictive or therapeutic dependence, and that benzodiazepines were more associated with the latter, not the former (Salzman 1991). Unfortunately, this nuanced approach to benzodiazepine-induced dependence was quickly lost in the noise produced by the more vocal and influential benzodiazepine opponents.

Concerns about benzodiazepine dependence made it much easier for the newer antidepressants to be promoted for the treatment of anxiety disorders (Starcevic 2011). As a result, by the end of the 1990s, most guidelines endorsed selective serotonin reuptake inhibitors (SSRIs) and serotonin and noradrenaline reuptake inhibitors (SNRIs) as the pharmacological treatment of choice for anxiety disorders. However, in 1999, one panel of world experts still recommended benzodiazepines for anxiety disorders, even for prolonged periods of time (Uhlenhuth 1999).

Studies confirm that benzodiazepines remain the most commonly used medications for anxiety disorders in the USA (55-94\% of patients with anxiety disorders were treated with benzodiazepines (Stahl 
2002)), despite efforts to promote antidepressants as first-line pharmacological treatment for these conditions (Bruce 2003; Vasile 2005). Frequent use of benzodiazepines has also been reported in several European countries (Smolders 2007; Demyttenaere 2008).

There are several reasons for the continuing popularity of benzodiazepines (Box 1), including their consistent effectiveness for relieving anxiety, tension and various physical symptoms of anxiety; quick onset of therapeutic action; relatively good tolerability; possibility of administration on an 'as-needed' (p.r.n.) basis; and relative safety in overdose. Problems with antidepressants in the treatment of anxiety disorders have also contributed to the continuing attractiveness of benzodiazepines. The following case vignette illustrates a common clinical scenario.

\section{A typical clinical case vignette}

A 56-year-old woman started taking diazepam 3 years ago because of panic attacks and anxiety This was at about the time she was going through a difficult divorce. Her mood has fluctuated over the years and she has had at least two episodes of depression. Treatment with several antidepressants failed because of intolerable side-effects: she was 'sick in the stomach', 'zonked out' or felt like she was going to 'explode'. At the time of referral, she was on a stable regimen of diazepam $15 \mathrm{mg} /$ day, and occasionally $20 \mathrm{mg} /$ day. She felt that diazepam helped her to deal with her financial worries and cope better with her adult children. She also believed that diazepam made it possible for her to sleep and relax. She denied experiencing any side-effects and was able to find a new job. At the assessment, she exhibited no significant symptoms of anxiety and she did not feel depressed. There was no history of substance misuse problems. She believed that ceasing diazepam would be premature, but was willing to reconsider it in the future.

\section{Mechanism of action, clinical effects and types of benzodiazepines}

It is widely believed that benzodiazepines enhance the effects of the neurotransmitter gammaaminobutyric acid (GABA). They do so through action at benzodiazepine receptors. Alleviation of fear is a result of GABA-induced inhibition of neuronal transmission that occurs in the amygdala, located in the brain stem, and thought to mediate brain circuits involved in the appraisal of threat and experience of fear.

Benzodiazepines produce a calming effect, decrease anxiety, relax tense muscles, alleviate symptoms of fear-related bodily arousal (e.g. heart racing, trembling), and promote sleep. It appears that soon after taking a benzodiazepine, many people experience a pleasant 'glowing' feeling
BOX 1 Reasons for benzodiazepine use in anxiety disorders

- Consistent effectiveness for relieving anxiety, tension, sleep disturbance and various physical symptoms of anxiety (particularly apparent in acute settings and when there is a need for quick alleviation of anxiety and distress)

- Quick onset of anti-anxiety effect (often within minutes of administration)

- Relatively good tolerability (side-effects rarely appear to be a reason for discontinuation)

- Possibility of administration on an 'as-needed' (p.r.n.) basis, which may be useful in a variety of settings

- Relative safety in overdose (especially if not taken in combination with other medications)

- Some disappointment with antidepressants in the treatment of anxiety disorders (their slow onset of therapeutic action, unpredictable and occasionally troublesome side-effects, inconsistent or unreliable effectiveness in some cases)

and a sense of remoteness from or indifference to their worries or fears, but it is unclear whether these effects play a significant role in motivating people with anxiety disorders to continue taking benzodiazepines. Perhaps the key characteristic of benzodiazepines is that when dosed correctly, they calm and relax without causing drowsiness, and therefore do not interfere with routine, everyday activities and functioning.

Box 2 lists commonly used benzodiazepines. The similarities between benzodiazepines are greater than their differences, and the main characteristic that distinguishes them is the duration of their action. They are usually divided into shortacting (up to $6 \mathrm{~h}$ ), intermediate-acting (6-12h) and

B0X 2 Commonly used benzodiazepines

\section{Short-acting}

- Alprazolam

- Lorazepam

Intermediate-acting

- Oxazepam

Long-acting

- Diazepam

- Ethyl loflazepate

- Clorazepate

- Chlordiazepoxide

- Clonazepam 
long-acting (more than $12 \mathrm{~h}$ ), although this division is not very clear. However, classification on these grounds has several practical implications.

Short-acting benzodiazepines (e.g. alprazolam) need to be taken three to four times a day, which is not very practical. For this reason, they may not be suitable for long-term administration. They may be more useful for immediate, one-off alleviation of anxiety or distress and may thus be a good option for p.r.n. use. These benzodiazepines should probably be avoided if there are concerns about misuse, because of an association, albeit inconclusive (e.g., Pradel 2010), between short duration of action and propensity for misuse.

Intermediate-acting (e.g. oxazepam) and longacting benzodiazepines (e.g. diazepam, clonazepam) should ideally be taken once or twice a day and they are usually preferred for long-term treatment. Long-acting benzodiazepines may accumulate, especially when the next dose is taken before the previous doses have been eliminated from the body. This may cause unexpected and excessive drowsiness ('hangover sedation'), especially in the elderly. As an intermediate-acting drug, oxazepam is therefore preferred by regulatory authorities in some countries.

\section{Benzodiazepines $v$. antidepressants for anxiety disorders}

Separating short-term and long-term treatment of anxiety disorders is somewhat artificial. Most anxiety disorders have a chronic course and the initial choice of pharmacological agent is likely to determine treatment over the subsequent months or even years. Thus, it is important to ascertain when treatment with benzodiazepines is a reasonable initial strategy (Box 3).

B0X 3 When to consider benzodiazepines for anxiety disorders

Clinical situations in which benzodiazepines may be considered as the initial treatment for anxiety disorders (with or without an antidepressant) include:

- panic disorder (and to a lesser extent, generalised anxiety disorder and social anxiety disorder)

- need for rapid symptomatic relief (e.g. for severe anxiety and frequent panic attacks)

- history of severe side-effects of the previously administered antidepressant(s), especially if these side-effects resulted in premature cessation of the medication

- absence of current or past substance misuse
Clinicians should first consider the principal cross-sectional diagnosis and whether benzodiazepines may be an effective treatment. The efficacy of benzodiazepines (especially alprazolam and clonazepam) in treating panic disorder is most convincing (e.g. Cross-National Collaborative Panic Study 1992; Rosenbaum 1997). Efficacy data are less consistent for generalised anxiety disorder and social anxiety disorder (e.g. Gelernter 1991; Davidson 1993; Rickels 1993, 2000), and benzodiazepines may be useful only in their short-term treatment and/or in conjunction with antidepressants. Benzodiazepines should ideally be used only as an adjunct in patients with post-traumatic stress disorder (PTSD), to target specific and particularly distressing symptoms for a limited period of time. They are not effective in obsessive-compulsive disorder (OCD) and specific phobias.

Benzodiazepines are most useful when there is need for rapid symptomatic relief - that is, when patients cannot tolerate distress caused by the severe physical symptoms and accompanying catastrophic thoughts and overwhelming anxiety which often occur as part of panic attacks in panic disorder and situational anxiety in social anxiety disorder. Also, they may quickly alleviate restlessness, improve sleep and decrease tension intensity in patients with generalised anxiety disorder.

Another factor that favours benzodiazepines is the relatively favourable side-effect profile and tolerability. It has been reported that people with anxiety disorders tend to tolerate benzodiazepines better than antidepressants (Schweizer 1993; Cowley 1997). If a patient has developed severe side-effects to an antidepressant in the past, especially if these side-effects led to premature discontinuation of that medication, beginning treatment with a benzodiazepine might be a logical choice. Most troublesome side-effects of antidepressants in this regard include increased anxiety and agitation ('jitteriness syndrome'), insomnia, and sexual dysfunction.

Antidepressants have several advantages over benzodiazepines (Box 4). Unlike antidepressants, benzodiazepines are ineffective in the treatment of depression and unsuitable as monotherapy for patients with anxiety disorders and a co-occurring depressive illness or a history of depression, both of which are frequently encountered in patients with generalised anxiety disorder and social anxiety disorder. In addition, antidepressants tend to have a greater effect on the key cognitive aspects of generalised anxiety disorder, such as pathological worry. Benzodiazepines should generally be 
BOX 4 When to consider antidepressants for anxiety disorders

Clinical situations in which antidepressants are more likely to be considered as the initial treatment for anxiety disorders include

- post-traumatic stress disorder, obsessive-compulsive disorder

- generalised anxiety disorder, social anxiety disorder (possibly in combination with a benzodiazepine)

- presence of a co-occurring depressive disorder or history of depression

- predominance of cognitive aspects of pathological anxiety (e.g. pathological worry)

- current or past alcohol or other substance misuse/ dependence, concern about benzodiazepine dependence and misuse

avoided if current or past substance use disorder is an issue, which may be particularly common in patients with social anxiety disorder. All these factors further suggest that benzodiazepines are less likely to be appropriate as monotherapy for the initial treatment of generalised anxiety disorder and social anxiety disorder than they may be for the initial treatment of panic disorder.

The strategy of commencing treatment with a low-dose antidepressant and a benzodiazepine to produce quick alleviation of distress, counteract initial agitation/increase in anxiety and bring about an earlier response has received some support, especially in panic disorder (Goddard 2001; Pollack 2003). Ideally, the benzodiazepine should be gradually discontinued after $6-10$ weeks, and treatment should continue with the antidepressant alone. In practice, this is not always easy. Some patients are reluctant to stop the benzodiazepine, especially if they have improved substantially and they attribute improvement to the benzodiazepine, not the antidepressant. Trying to convince the patient that stopping the benzodiazepine is the only 'right' thing to do may not be the best course to take. Other patients experience some worsening of symptoms while the benzodiazepine dose is being reduced and insist on continued treatment for that reason. Therefore, some patients continue taking both medications for prolonged periods.

\section{Issues in long-term benzodiazepine use}

As a long-term treatment for anxiety disorders, benzodiazepines will be administered either as monotherapy or in combination with an antidepressant. Benzodiazepines should be continued for at least 6-12 months after remission has been attained. To maximise treatment effects, patients need to be given accurate and unambiguous information about the benefits and risks of longterm benzodiazepine use.

Several issues have been raised in relation to the long-term treatment of anxiety disorders with benzodiazepines (Table 1), and most of them have become seldom-challenged myths: patients on long-term treatment develop tolerance and benzodiazepines thus lose efficacy and their doses have to be increased to maintain benefit; patients crave for benzodiazepines, which leads to misuse and addiction; benzodiazepines cannot be stopped because withdrawal symptoms are too dangerous; patients may develop debilitating sideeffects, especially severe cognitive impairment. In short, the misconception is that long-term benzodiazepine use is dangerous and inevitably leads to misuse and addiction.

Despite these myths, there is a relative paucity of studies of long-term benzodiazepine use in patients with anxiety disorders. The myths and evidence will be discussed, with the goal of providing a more balanced view of the benefits and risks of long-term benzodiazepine use. It should be noted that research findings have implications only for patients with an anxiety disorder who do not have a propensity for substance use disorders and who do not have a personality disturbance (e.g. borderline, antisocial or dependent personality disorders) that might make it more difficult for them to cease benzodiazepine use.

\section{Tolerance}

There have been reports of patients who needed to increase the dose of benzodiazepines to continue experiencing the initial anti-anxiety effect.

\section{TABLE 1 Common misconceptions about long-term benzodiazepine treatment of anxiety disorders}

\begin{tabular}{|ll|}
\hline Misconception & Status/evidence \\
\hline $\begin{array}{l}\text { Tolerance to the } \\
\text { anxiolytic effect }\end{array}$ & $\begin{array}{l}\text { Vast majority of patients with anxiety disorders do not show } \\
\text { a tendency to increase the dose and do not exhibit loss of the } \\
\text { therapeutic benefit }\end{array}$ \\
\hline Frequent misuse & $\begin{array}{l}\text { Benzodiazepine misuse rarely occurs in patients with anxiety } \\
\text { disorders who do not have current or past substance misuse problems }\end{array}$ \\
\hline Frequent addiction & $\begin{array}{l}\text { Substance addiction and benzodiazepine dependence are different } \\
\text { phenomena, with little overlap }\end{array}$ \\
\hline $\begin{array}{l}\text { Dangerous withdrawal } \\
\text { symptoms }\end{array}$ & $\begin{array}{l}\text { Withdrawal symptoms may occur in the course of benzodiazepine } \\
\text { taper and are very likely to occur on abrupt discontinuation, but they } \\
\text { are rarely dangerous; the risk of withdrawal should be minimised } \\
\text { through a gradual and individualised taper }\end{array}$ \\
\hline Debilitating side-effects & $\begin{array}{l}\text { Benzodiazepines are generally well tolerated and there is no evidence } \\
\text { that they cause organ toxicity; their side-effects, especially sedation } \\
\text { and impairment of motor coordination, should be closely monitored }\end{array}$ \\
\hline
\end{tabular}


However, several studies (Nagy 1989; Pollack 1993; Worthington 1998; Soumerai 2003) have shown that during long-term treatment with benzodiazepines, the vast majority of patients do not have a tendency to increase the dose. Three of these studies (Nagy 1989; Pollack 1993; Worthington 1998) also reported no loss of therapeutic benefit of clonazepam and alprazolam in the course of long-term (up to 2.5 years) treatment, suggesting that there was no tolerance to the anxiolytic effects of these drugs.

\section{Dependence, misuse and addiction}

\section{Dependence}

Patients with anxiety disorders treated continuously with benzodiazepines for several weeks develop therapeutic or non-addictive dependence. This dependence is pharmacological (physical) in nature; it is a consequence of the physiological adaptation at the receptor level to the continuous use of benzodiazepines (Haefely 1986). The main implication of therapeutic dependence is that people using benzodiazepines for a long time should not cease them abruptly because of the likelihood of withdrawal symptoms; therapeutic dependence does not mean misuse, drug-seeking or lack of benefit (Rosenbaum 2005). It is crucial to distinguish therapeutic benzodiazepine dependence from benzodiazepine misuse and dependence related to true addiction.

\section{Misuse}

Benzodiazepine misuse is a pattern of indiscriminate use with harmful behaviour (e.g. stealing to obtain the medication), often with a tendency to increase the dose. In the absence of a history of alcohol or other substance misuse or dependence, benzodiazepine misuse among patients with anxiety disorders is rare (Salzman 1991; Salzman 1993; Andersch 2003). Patients

TABLE 2 Comparison between benzodiazepine dependence occurring in the course of long-term use for anxiety disorders and substance addiction

\begin{tabular}{|lcc|}
\hline & $\begin{array}{c}\text { Non-addictive/therapeutic } \\
\text { benzodiazepine } \\
\text { dependence }\end{array}$ & $\begin{array}{c}\text { Substance } \\
\text { addiction }\end{array}$ \\
\hline Tolerance & No & Yes \\
\hline $\begin{array}{l}\text { An all-encompassing preoccupation with and/ } \\
\text { or craving for the substance }\end{array}$ & No & Yes \\
\hline Uncontrollable drug-seeking behaviour & No & Yes \\
\hline $\begin{array}{l}\text { Adverse health and/or social consequences } \\
\text { (e.g. infection, liver disease, damage to one's } \\
\text { reputation, financial and legal problems) }\end{array}$ & No & Yes \\
\hline $\begin{array}{l}\text { Withdrawal symptoms on abrupt } \\
\text { discontinuation }\end{array}$ & Yes & \\
\hline
\end{tabular}

with anxiety disorders on long-term treatment who have developed physical dependence are not misusing benzodiazepines (O'Brien 2005). Likewise, patients with anxiety disorders who have no tendency to misuse alcohol or other drugs, usually need not worry that they will end up misusing benzodiazepines.

\section{Addiction}

Addiction encompasses an intense craving for and preoccupation with a substance, uncontrollable drug-seeking behaviour, tolerance, adverse health and/or social consequences, and occurrence of withdrawal symptoms on abrupt discontinuation of the substance (Table 2). Of these characteristics, benzodiazepines are only associated with withdrawal symptoms on abrupt discontinuation. Although craving for benzodiazepines has been described, it occurs mainly in the context of craving for other substances or as a consequence of personality-level psychopathology (e.g. Mol 2005).

\section{Pharmacological $v$. psychological dependence}

Distinction also needs to be made between pharmacological and psychological dependence. Although all people taking benzodiazepines for prolonged periods of time become pharmacologically dependent, only some develop psychological dependence. The latter refers to the manifestations of dependence that are not based on the pharmacological actions of benzodiazepines. For example, people who 'must' carry their benzodiazepine wherever they go, may believe that the medication prevents anxiety in some magical way. This is sometimes referred to as 'talisman dependence' and is a good example of the use of medication as a safety device. Another type of psychological dependence occurs in people who have been gradually decreasing the dose to the point of taking a minimal dose, but then they are unable to complete the taper ('lastdose dependence'). This is a consequence of the protective role attributed to the medication and patients' unwillingness to face anxiety without relying on the medication.

\section{Withdrawal symptoms and return of anxiety after cessation of benzodiazepines}

Benzodiazepine withdrawal syndrome is often portrayed as dangerous and it is therefore dreaded by both patients and physicians. In fact, some patients continue taking benzodiazepines only to avoid withdrawal. This intensifies their fear that they will not be able to stop the medication and reinforces the notion that benzodiazepines are addictive. 
Most withdrawal symptoms are not specific and resemble a recurrence of an anxiety disorder (Box 5). Relatively specific symptoms can help in recognising withdrawal syndrome, but they are not always present. Seizures are the most serious feature of benzodiazepine withdrawal, but they are rare. Withdrawal symptoms are rarely life threatening, they do not last very long (from several days to 2-3 weeks), usually leave no consequences, and often disappear without the need for treatment.

\section{Tapering medication}

Benzodiazepine withdrawal syndrome should be neither overestimated nor trivialised. The likelihood of withdrawal should be minimised through a very gradual and individualised tapering schedule before the medication is stopped entirely. Depending on the dose, the type of benzodiazepine (short-acting, intermediate- or long-acting), duration of its use, and the patient's overall condition and personality characteristics, the taper can be completed in weeks or over a period of many months. As an example, a decrease in the total daily dosage by $0.25 \mathrm{mg}$ of alprazolam over $1-2$ weeks or by $0.125-0.25 \mathrm{mg}$ of clonazepam over 1-2 weeks should be adequate to minimise the risk of withdrawal symptoms for most patients.

Patients need to feel ready for benzodiazepine discontinuation in order for it to be successful. In other words, they need to indicate that they feel capable of coping with the anxiety without having to continuously rely on the medication. If patients resist cessation, it is a bad idea to antagonise them by insisting that they stop 'no matter what'. Refusing to continue prescribing a benzodiazepine or using other coercive measures is only likely to lead to more difficulties. It is also important to be flexible about the duration of taper, for example, by not setting rigid time limits.

The taper should ideally proceed under the physician's supervision because they are best placed to suggest the pace of decreasing the dose, to monitor the patient's progress and to provide support. The last is especially important if withdrawal symptoms occur during taper or if patients become ambivalent about continuing the taper. Cognitive-behavioural therapy (CBT) may also be effective in facilitating benzodiazepine discontinuation (Otto 1993; Spiegel 1994).

\section{Rebound and relapse}

Rebound refers to the return of anxiety symptoms within several days of benzodiazepine cessation. These symptoms resemble the manifestations of anxiety before the onset of treatment, but they
B0X 5 Characteristics of benzodiazepine withdrawal syndrome

\begin{tabular}{ll}
\hline Non-specific symptoms & Specific symptoms \\
- Increased anxiety & - Hypersensitivity to light, sound, smell or \\
- Physical symptoms of anxiety & taste \\
- Restlessness & - Feeling of things moving, as if being on \\
- Sleep disturbance & a boat \\
- Nausea, stomach cramps & - Tinnitus \\
- Numbness or tingling sensations & - Distorted body image \\
- Unsteady gait & - Muscle cramps and pains, myoclonic jerks \\
- Irritability & - Flu-like symptoms \\
- Feeling depressed & - Suspiciousness \\
- Feeling weak & - Feeling confused \\
- Tiredness & - Depersonalisation, derealisation \\
& - Seizures (rarely)
\end{tabular}

are more severe. It may be difficult to distinguish rebound from withdrawal syndrome.

A full-blown relapse, usually occurring several weeks or months after medication cessation, is another possible outcome of benzodiazepine discontinuation. There is some indication that relapse rates may be higher after cessation of benzodiazepines than after cessation of antidepressants (Noyes 1991).

Both rebound and relapse may call for recommencement of the original pharmacotherapy, consideration of a different pharmacological approach, or a combination of pharmacological and psychological interventions. Adding CBT to ongoing pharmacotherapy may decrease the risk of relapse by introducing effective anxiety management techniques, fostering a more active approach to treatment, and improving coping and self-efficacy (Biondi 2003).

\section{Side-effects}

\section{Sedation}

Benzodiazepines are generally well tolerated. Their most common side-effect is sedation. For the most part, sedation is dose dependent, so that the higher the dose, the more likely it is for people to feel slowed down, tired, drowsy or even sleepy. Sedation typically occurs at the very beginning of treatment or immediately after the dose has been increased. Most people develop tolerance to the sedative effects of benzodiazepines, and the initial dose that caused sedation ceases to do so within 1-2 weeks of continued administration (Shader 1993). As a result, sedation usually disappears and is generally not a problem for patients on a stable dose of medication. Unless sedative effects persist, the dose need not be decreased. 


\section{Psychomotor impairment}

Another common and potentially troublesome side-effect is interference with psychomotor performance. This may affect complex skills such as driving (e.g. Rapoport 2009), and a link between benzodiazepine use and motor vehicle accidents has been suggested (e.g. Barbone 1998). This interference requires careful monitoring, especially during the initial stages of treatment and while the dose is being increased. However, calls to limit prescription of benzodiazepines because of their possible interference with driving have not been widely supported, as some studies failed to relate long-term benzodiazepine use to psychomotor impairment (e.g. Lucki 1986).

It has also been suggested that no dose or serum level of a benzodiazepine has been clearly associated with impairment of driving ability (Freeman 2009) and that limiting benzodiazepine prescription 'across the board' is not warranted for this reason. Nevertheless, impairment of motor coordination is dose dependent, so patients should not drive or operate machines immediately after a dose increase.

\section{Use with elderly patients}

Excessive sedation and impaired psychomotor performance may be particularly troublesome in the elderly, as this may lead to falls and fractures. Although it has not been confirmed that hip fracture rates in the elderly are necessarily associated with benzodiazepine use (Wagner 2007), elderly patients should use benzodiazepines with caution and at the lowest possible dose.

Interactions with alcohol

People taking benzodiazepines should avoid alcohol, because the combination of alcohol and benzodiazepines intensifies the sedative effects of the drug and may further impair motor coordination.

\section{Amnesia}

Anterograde amnesia occurs relatively frequently and refers to a difficulty remembering what happened in the period of up to several hours after a benzodiazepine has been taken. In most cases, memory can still be retrieved, although with some effort. This side-effect can be disturbing, especially to patients who need to remember what they have learnt in the period after taking a benzodiazepine.

Disinhibition

Disinhibition, manifested through irritability, anger and/or behaviour that is inappropriate, 'out of character' or aggressive, is not a common sideeffect (Rothschild 2000). Still, individuals with emotionally unstable personalities, immaturity, impulse control problems, brain damage and substance misuse may be more likely to exhibit disinhibition, and it may be prudent to avoid benzodiazepines in these circumstances.

\section{Cognitive abnormalities}

There are conflicting views on the association of benzodiazepines with cognitive abnormalities. A meta-analytic study of the cognitive effects of long-term benzodiazepine use (Barker 2004), suggesting a significant decline in all cognitive domains, had serious methodological flaws. One brain imaging study (Busto 2000) did not find benzodiazepine-induced brain abnormalities that could underlie cognitive changes. Significant cognitive impairment is rarely observed in the course of long-term benzodiazepine treatment of anxiety disorders (Deckersbach 2011), but subtle changes may occur, calling for further research.

\section{Depression}

Although it is occasionally reported that benzodiazepines cause depression or make depressive symptoms worse, there is no conclusive evidence that these drugs are depressogenic.

\section{Ensuring effective and safe use of benzodiazepines}

Benzodiazepines are not ideal drugs for treating anxiety disorders. Like other pharmacological agents, they have advantages and disadvantages, but the latter have been emphasised at the expense of the former. Considering that a need for pharmacological management of anxiety disorders is not likely to disappear, it is time to reappraise the role of benzodiazepines in the treatment of anxiety disorders and make recommendations for their effective and safe use (Box 6).

First, there should be careful consideration of the type of patient who might benefit from benzodiazepines, for example, a person with panic disorder. These medications are not effective in PTSD and OCD and they are generally not very useful in generalised anxiety disorder and social anxiety disorder if administered alone. People with current or past substance misuse problems and those with severe personality disorder are generally unsuitable for treatment with benzodiazepines because of their propensity to misuse agents with dependence-producing capability.

Second, the administration of benzodiazepines should be considered in clinical situations in which 
BOX 6 Measures to ensure safe, effective use of benzodiazepines for anxiety disorders

- Careful selection of patients who might benefit from benzodiazepines

- Administration of benzodiazepines in clinical situations in which they are more likely to be useful (see Box 3)

- If possible, administration in a lower dose, for a short period of time, and in conjunction with an antidepressant

- Careful monitoring and management of side-effects

- Right 'timing' for benzodiazepine cessation

- Careful, gradual and individualised benzodiazepine taper to prevent or alleviate withdrawal symptoms

they are most likely to be useful. These include presence of severe symptoms of anxiety and tension, frequent and severe panic attacks, sleep disturbance, restlessness and agitation, especially when patients have great difficulty tolerating these symptoms and request immediate relief.

Third, benzodiazepines should ideally be administered in a lower dose, for a short time (e.g. 6-10 weeks) and in conjunction with an antidepressant. However, long-term monotherapy with benzodiazepines is a viable and justified option.

Fourth, side-effects of benzodiazepines should be carefully monitored and managed (e.g. by adjusting the dosage accordingly).

Finally, benzodiazepine cessation should be considered only when the patient is ready. Once the decision has been made to discontinue, this should be done carefully, gradually and in close collaboration with the physician to minimise the risk of withdrawal syndrome.

\section{References}

Andersch S, Hetta J (2003) A 15-year follow-up study of patients with panic disorder. European Psychiatry 18: 401-8.

Balter MB, Levine J, Manheimer D (1974) Cross-national study of the extent of anti-anxiety/sedative drug use. New England Journal of Medicine 290: 769-74.

Barbone F, McMahon AD, Davey PG, et al (1998) Association of roadtraffic accidents with benzodiazepine use. Lancet 352: 1331-6.

Barker MJ, Greenwood KM, Jackson M, et al (2004) Cognitive effects of long-term benzodiazepine use: a meta-analysis. CNS Drugs 18: 37-48.

Biondi M, Picardi A (2003) Increased probability of remaining in remission from panic disorder with agoraphobia after drug treatment in patients who received concurrent cognitive-behavioural therapy: a follow-up study. Psychotherapy and Psychosomatics 72: 34-42.

Bruce SE, Vasile RG, Goisman RM, et al (2003) Are benzodiazepines still the medication of choice for patients with panic disorder with or without agoraphobia? American Journal of Psychiatry 160: 1432-8.

Busto UE, Bremner KE, Knight K, et al (2000) Long-term benzodiazepine therapy does not result in brain abnormalities. Journal of Clinical Psychopharmacology 20: 2-6.
Cowley DS, Ha EH, Roy-Byrne PP (1997) Determinants of pharmacologic treatment failure in panic disorder. Journal of Clinical Psychiatry 58: 555-61.

Cross-National Collaborative Panic Study, Second Phase Investigators (1992) Drug treatment of panic disorder. Comparative efficacy of alprazolam, imipramine, and placebo. British Journal of Psychiatry 160 : 191-202.

Davidson JRT, Petts N, Richichi E, et al (1993) Treatment of social phobia with clonazepam and placebo. Journal of Clinical Psychopharmacology 13: $423-8$.

Deckersbach T, Moshier SJ, Tuschen-Caffier B, et al (2011) Memory dysfunction in panic disorder: an investigation of the role of chronic benzodiazepine use. Depression and Anxiety 28: 999-1007.

Demyttenaere K, Bonnewyn A, Bruffaerts R, et al (2008) Clinical factors influencing the prescription of antidepressants and benzodiazepines: results from the European study of the epidemiology of mental disorders (ESEMeD). Journal of Affective Disorders 110: 84-93.

Freeman SA (2009) The benzodiazepine stigma persists. Journal of Clinical Psychiatry 70: 1727-8.

Gelernter CS, Unde TW, Cimbolic P, et al (1991) Cognitive-behavioral and pharmacological treatments of social phobia: a controlled study. Archives of General Psychiatry 48: 938-45.

Goddard AW, Brouette T, Almai A, et al (2001) Early coadministration of clonazepam with sertraline for panic disorder. Archives of General Psychiatry 58: 681-6.

Haefely W (1986) Biological basis of drug-induced tolerance, rebound, and dependence: contribution of recent research on benzodiazepines. Pharmacopsychiatry 19: 353-61.

Healy D (2002) Psychiatric Drugs Explained. Third Edition: 142. Churchill Livingstone.

Lucki I, Rickels K, Geller AM (1986) Chronic use of benzodiazepines and psychomotor and cognitive test performance. Psychopharmacology (Berlin) 88: 426-33.

Mol AJJ, Gorgels WJMJ, Voshaar RCO, et al (2005) Associations of benzodiazepine craving with other clinical variables in a population of general practice patients. Comprehensive Psychiatry 46: 353-60.

Nagy LM, Krystal JH, Woods SW, et al (1989) Clinical and medication outcome after short-term alprazolam and behavioral group treatment in panic disorder: 2.5 year naturalistic follow-up study. Archives of General Psychiatry 46: 993-9.

Noyes R, Garvey MJ, Cook B (1991) Controlled discontinuation of benzodiazepine treatment for patients with panic disorder. American Journal of Psychiatry 148: 517-23.

O'Brien CP (2005) Benzodiazepine use, abuse, and dependence. Journal of Clinical Psychiatry 66 (suppl 2): 28-33.

Otto MW, Pollack MH, Sachs GS, et al (1993) Discontinuation of benzodiazepine treatment: efficacy of cognitive-behavioral therapy for patients with panic disorder. American Journal of Psychiatry 150: 1485-90.

Pollack MH, Otto MW, Tesar GE, et al (1993) Long-term outcome after acute treatment with alprazolam or clonazepam for panic disorder. Journal of Clinical Psychopharmacology 13: 257-63.

Pollack MH, Simon NM, Worthington JJ, et al (2003) Combined paroxetine and clonazepam treatment strategies compared to paroxetine monotherapy for panic disorder. Journal of Psychopharmacology 17: 276-82.

Pradel V, Delga C, Rouby F, et al (2010) Assessment of abuse potential of benzodiazepines from a prescription database using "doctor shopping" as an indicator. CNS Drugs 24: 611-20.

Rapoport MJ, Lanctot KL, Streiner DL, et al (2009) Benzodiazepine use and driving: a meta-analysis. Journal of Clinical Psychiatry 70: 663-73.

Rickels K, Downing R, Schweizer E, et al (1993) Antidepressants for the treatment of generalized anxiety disorder: a placebo-controlled comparison of imipramine, trazodone, and diazepam. Archives of General Psychiatry 50: 884-95.

\section{MCO answers \\ $1 c \quad 2 b \quad 3$ e $4 c \quad 5 b$}


Rickels K, DeMartinis N, Aufdembrinke B (2000) A double-blind, placebocontrolled trial of abecarnil and diazepam in the treatment of patients with generalized anxiety disorder. Journal of Clinical Psychopharmacology 20: 12-8.

Rosenbaum JF, Moroz G, Bowden CL (1997) Clonazepam in the treatment of panic disorder with or without agoraphobia: a dose-response study of efficacy, safety, and discontinuance. Journal of Clinical Psychopharmacology 17: 390-400

Rosenbaum JF (2005) Attitudes toward benzodiazepines over the years. Journal of Clinical Psychiatry 66 (suppl 2): 4-8.

Rothschild AJ, Shindul-Rothschild J, Viguera A, et al (2000) Comparison of the frequency of behavioral disinhibition on alprazolam, clonazepam, or no benzodiazepine in hospitalized psychiatric patients. Journal of Clinical Psychopharmacology 20: 7-11.

Salzman C (1991) The APA Task Force report on benzodiazepine dependence, toxicity, and abuse. American Journal of Psychiatry 148: 151-2.

Salzman C, Miyawaki EK, le Bars P, et al (1993) Neurobiologic basis of anxiety and its treatment. Harvard Review of Psychiatry 1: 197-206.

Schweizer E, Rickels K, Weiss S, et al (1993) Maintenance drug treatment of panic disorder: I. Results of a prospective, placebo-controlled comparison of alprazolam and imipramine. Archives of General Psychiatry 50: 51-60.

Shader RI, Greenblatt DJ (1993) Use of benzodiazepines in anxiety disorders. New England Journal of Medicine 328: 1398-405.

Smolders M, Laurant M, van Rijswijk E, et al (2007) The impact of comorbidity on GPs' pharmacological treatment decisions for patients with an anxiety disorder. Family Practice 24: 538-46.
Soumerai SB, Simoni-Wastila L, Singer C, et al (2003) Lack of relationship between long-term use of benzodiazepines and escalation to high dosages. Psychiatric Services 54: 1006-11.

Spiegel DA, Bruce TJ, Gregg SF, et al (1994) Does cognitive behavior therapy assist slow-taper alprazolam discontinuation in panic disorder? American Journal of Psychiatry 151: 876-81.

Stahl SM (2002) Don't ask, don't tell, but benzodiazepines are still the leading treatments for anxiety disorder. Journal of Clinical Psychiatry 63 : 756-7.

Starcevic V (2011) Have anxiety disorders been disowned by psychiatrists? Australasian Psychiatry 19: 12-6.

Uhlenhuth EH, Balter MB, Ban TA, et al (1999) International study of expert judgment on therapeutic use of benzodiazepines and other psychotherapeutic medications: IV. therapeutic dose dependence and abuse liability of benzodiazepines in the long-term treatment of anxiety disorders. Journal of Clinical Psychopharmacology 19 (suppl 2): 23-29s

Vasile RG, Bruce SE, Goisman RM, et al (2005) Results of a naturalistic longitudinal study of benzodiazepine and SSRI use in the treatment of generalized anxiety disorder and social phobia. Depression and Anxiety 22: 59-67.

Wagner AK, Ross-Degnan D, Gurwitz JH, et al (2007) Effect of New York State regulatory action on benzodiazepine prescribing and hip fracture rates. Annals of Internal Medicine 146: 96-103.

Worthington JJ, Pollack MH, Otto MW, et al (1998) Long-term experience with clonazepam in patients with a primary diagnosis of panic disorder. Psychopharmacology Bulletin 34: 199-205.

\section{MCQs}

Select the single best option for each question stem

1 Reasons for the ongoing popularity of benzodiazepines in the treatment of anxiety disorders include:

a their greater effectiveness than antidepressants

b their effectiveness against the cognitive symptoms of anxiety (e.g. pathological worry)

c quick onset of anti-anxiety effect

d their effectiveness for all anxiety disorders

e favourable effect on co-occurring depressive illness.

\section{Clinical situations in which benzo-} diazepines may be considered as the initial treatment for anxiety disorders include:

a presence of OCD

b presence of severe panic attacks and anxiety for which quick relief is requested

c presence of social anxiety disorder in conjunction with current alcohol misuse $d$ presence of generalised anxiety disorder and major depression

e presence of PTSD.

3 Characteristics of benzodiazepine dependence occurring in the course of their long-term use include:

a tolerance to the anxiolytic effects of benzodiazepines

b an all-encompassing preoccupation with and/or craving for benzodiazepines

c uncontrollable benzodiazepine-seeking behaviour

$d$ adverse health and/or social consequences

e withdrawal symptoms on abrupt discontinuation.

4 Relatively specific symptoms of benzodiazepine withdrawal syndrome include:

a restlessness

b tiredness

c hypersensitivity to light, sound, smell or taste d insomnia

e irritability.

5 Measures to ensure effective and safe use of benzodiazepines in anxiety disorders include:

a benzodiazepine cessation as soon as possible, even if the patient is not agreeable

b careful selection of patients who might benefit from benzodiazepines

c their use on an as-needed (p.r.n.) basis only

$d$ frequent testing of cognitive functioning, even in younger patients

e use of carbamazepine to prevent withdrawal symptoms. 